\title{
Classifying complex road features in the context of car driver education
}

\author{
Jukka M. Krisp ${ }^{a}$ *, Andreas Keler ${ }^{b}$ \\ ${ }^{a}$ University of Augsburg, Institute of Geography, Applied Geoinformatics, Alter Postweg 118, 86159 Augsburg, Germany, \\ jukka.krisp@geo.uni-augsburg.de \\ ${ }^{b}$ Technical University of Munich, Chair of Traffic Engineering and Control, Arcisstraße 21, 80333 Munich, Germany, \\ andreas.keler@tum.de \\ * Corresponding author
}

Keywords: Location Based Services (LBS), routing, car navigation, way finding, driving schools

\begin{abstract}
:
Learning to drive a car is not easy. Therefore, in many countries, driving schools offer an education of how to drive a car. This is taught on a theoretical and practical level. Once a driving student has learned how to handle a car in real traffic, he / she will take a driving test exam. In Germany, for example, an authorized driving examiner telling the students to follow their instructions conducts the practical exam. Thereby the driving examiner guides the student to different situation (for example taking a highway, crossing a complicated intersection, backward parking etc.). The examiner selects the route according to the criteria set out in the test guidelines and incorporates the road and traffic conditions in his choice. The driver test guidelines include basic driving tasks (parking, danger braking, etc.), a route within closed villages, a route outside built-up areas and / or motorway sections and afterwards a feedback on student performance. The test is set for a period of 45 minutes (for driving class B). Currently mobile devices, which are able to record a number of parameters (location, speed etc.) are rarely used in these driving examinations. Additionally, the route the driving instructor follows can be somewhat arbitrary. Utilizing routing systems in these driving examinations may assist the driving examiner and may help to make the examination more transparent.
\end{abstract}

How can utilize routing algorithms to support driving examinations? In general routing algorithms offer a number of different parameters that may provide users with different type of routes, including the shortest, fastest, safest, most beautiful, least fuel/energy consumption (Ranacher et al. 2016), male/female (Häusler et al. 2010), easiest (Duckham and Kulik 2003) or most difficult (to drive) route (Krisp, Keler, and Karrais 2014). As Krisp \& Keler (2015) suggest, that may also include the 'most difficult to drive route', which might be useful for driver training purposes. Previous research (Krisp and Keler 2015) has investigated what is an "easy to drive" route? and "what are the traffic situations that could be avoided for inexperienced drivers and/or driving beginners?". A number of commercial vendors offer products that include pre-programmed routes to help driving students to "learn" particular situations.

We investigate selected parameters relevant for a driving test, in particular complicated crossings. This requires examinations on specific situations and the complexity of the road infrastructure. In a wider context, we aim to provide suggestions for a routing system that will help driving instructors and driving examiners, use standardized routes and thereby make driving examinations more transparent.

Initial research in this context has been conducted an online questionnaire at the University of Augsburg. This questionnaire includes about seventy-five participants, which are mainly students. About $89 \%$ do have a driving license longer those two years. About $25 \%$ do not use a car on a regular base. Within this online questionnaire, sixteen driving situations are described. The participants rate each driving situation by agreeing or disagreeing to statements on these situations. For example, to the statement "I have problems with complicated crossings", about $43 \%$ of participants "strongly agreed" or "agreed". The questionnaire shows that the basis for what is in easy or difficult to drive route seems to vary, based on the individual driver.

A starting point to investigate, within the large number of driving situations, are "complicated crossings" (Krisp and Keler 2015) and to consider interactions to vulnerable road users. Figures 1 illustrates the challenge of defining complicated crossings based on static measures and dynamic measures. Static measures include the number of nodes that can be extracted from a road database, based on Krisp \& Keler (Krisp and Keler 2015). Dynamic measures, like the traffic density or interaction points (between the traffic participants) at urban crossings are acquired via analysis of extracted video trajectories from cameras. 


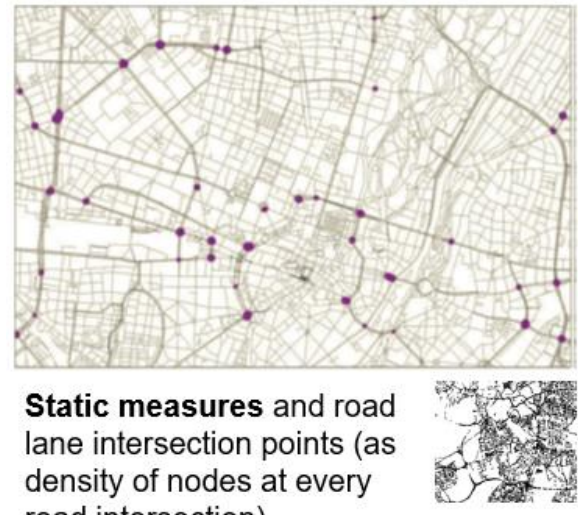

road intersection)

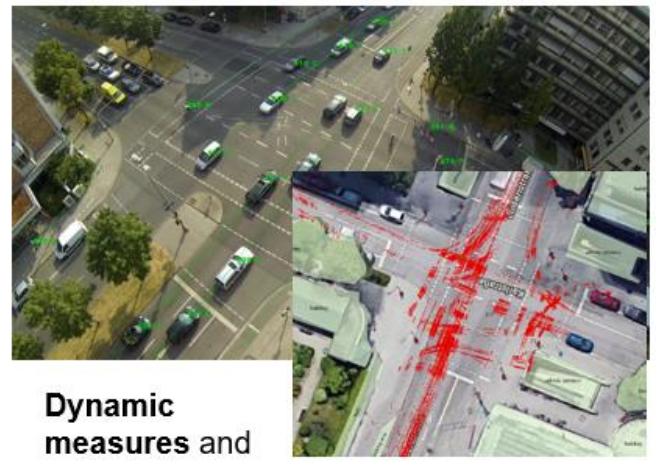

extracted

conflict points

Figure 1. Illustration showing the challenge of defining complicated crossings based on static measures (based on nodes derived from Open-Street Map) and dynamic measures (based on near real-time traffic cameras)

The complexity of an intersection is also dependent on the phases of the traffic signal system or the traffic signs. Initial data includes the most common types of intersections in Germany, in the context of the most frequently appearing accidents (as from previous analyses based on the GIDAS accident database focusing on the type of involved traffic participants, for example car-car, car-bicycle, car-pedestrian). These are divided into different node accident groups. Utilizing these parameters within the concept of "complicated crossings" gives a better idea of which situations might be "more difficult" or "less difficult" in the context of driving tests.

Expanding this model to other static parameters, like "left turns" (Winter 2002) or dynamic parameters, like "bicycles on the road" provides the option of computing "demanding" or "easy to drive" routes. Theoretically, we have a finite number of routes from A to B assuming a static network. Still, in this context, each route does have its own "difficulty index". This index changes depending on defined static and dynamic parameters also for every road segment (based for example on traffic, weather etc.), crossing and the personal preferences by a potential driving test candidate. As for further research, a difficulty is how to measure the personal "difficulty" / "easiness" to drive a route? That opens the question, is it possible to build "standardized" routes for driving test as they are "difficult" or "easy" based on the individual?

\section{References}

Duckham, M., and L. Kulik. 2003. '“Simplest” Paths: Automated Route Selection for Navigation.' in W. Kuhn, Worboys, M. F., and Timpf, S. (ed.), Spatial Information Theory. Foundations of Geographic Information Science, LNCS2825 (Springer: Berlin).

Häusler, E., R. Steinmann, G. Gartner, and M. Schmidt. 2010. "The FEMroute Project - A Gender-Sensitive Approach to Route Planning Systems for Pedestrians." In Proceedings of the 7th International Symposium on Location Based Services \& Tele Cartography", G. Gartner, Y. Li (ed.); (2010), 124 - 128.

Krisp, Jukka M., and Andreas Keler. 2015. 'Car Navigation - Computing Routes that Avoid Complicated Crossings', International Journal of Geographical Information Science, 29: 1988-2000.

Krisp, Jukka M., Andreas Keler, and Nicole Karrais. 2014. "Personalized Routing for Car Navigation Systems." In 11th International Symposium on Location-Based Services, edited by Georg Gartner and Haosheng Huang, 51-53. Vienna, Austria.

Ranacher, Peter, Richard Brunauer, Stefan Christiaan Van der Spek, and Siegfried Reich. 2016. 'A model to estimate and interpret the energy-efficiency of movement patterns in urban road traffic', Computers, Environment and Urban Systems, 59: 152-63.

Winter, S. 2002. 'Modeling costs of turns in route planning', Geoinformatica, 6: 345-61. 